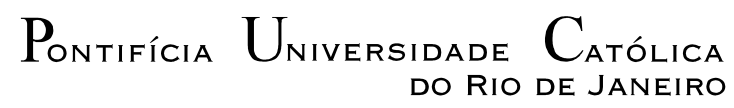
DO RIO DE JANEIRO

IRENE ELIAS RODRIGUES

\title{
A INCLUSÃO DE PESSOAS COM NECESSIDADES EDUCACIONAIS ESPECIAIS NO PROCESSO EDUCATIVO ESCOLAR: UMA EXPERIÊNCIA INVERSA.
}

Tese apresentada ao Programa de Pós-Graduação em Educação Brasileira da PUC-Rio como parte dos requisitos para a obtenção do título de Doutor em Educação Brasileira.

Orientadora: Profa . Isabell Alice Osvald Monteiro Lelis Co-Orientadora: Prof ${ }^{a}$. Ivanilde Apoluceno de Oliveira 


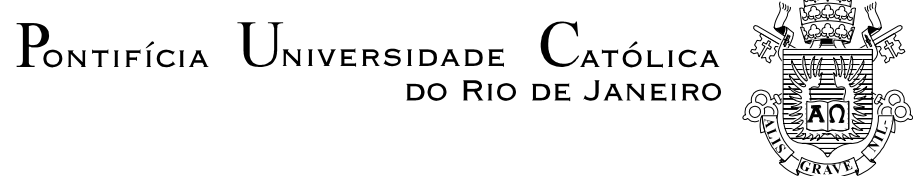

IRENE ELIAS RODRIGUES

\title{
A INCLUSÃO DE PESSOAS COM NECESSIDADES EDUCACIONAIS ESPECIAIS NO PROCESSO EDUCATIVO ESCOLAR: UMA EXPERIÊNCIA INVERSA.
}

\begin{abstract}
Tese apresentada como requisito parcial para obtenção do grau de Doutor pelo Programa de Pósgraduação em Educação do Departamento de Educação do Centro de Teologia e Ciências Humanas da PUC-Rio. Aprovada pela Comissão Examinadora abaixo assinada.
\end{abstract}

Profa. Isabel Alice Oswald Monteiro Lelis

Orientadora

Departamento de Educação - PUC-Rio

Prof ${ }^{a}$. Ivanilde Apoluceno de Oliveira

Co-Orientadora

Universidade do Estado do Pará

Prof ${ }^{\text {a }}$ Vera Maria Ferrão Candau

Departamento de Educação - PUC-Rio

Profa. Theresinha Guimarães Miranda

Universidade Federal da Bahia

Profa. Denise Meyrelles de Jesus Universidade Federal do Espírito Santo

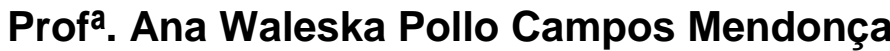

Departamento de Educação - PUC -Rio

Profa. Denise Berruezo Portinari

Coordenadora Setorial do Centro de Teologia e Ciências Humanas

PUC-Rio

Rio de Janeiro, 1 
Todos os direitos reservados. É proibida a reprodução total ou parcial do trabalho sem a autorização da universidade, do autor e do orientador.

Irene Elias Rodrigues

Irene Elias Rodrigues é Licenciado Pleno em Letras (Português-Francês), Mestre em Educação Docência Universitária pelo Instituto Pedagógico Latino Americano e Caribenho (IPLAC - República de Cuba -Havana). Foi bolsista do CNPq nas modalidades de Aperfeiçoamento em Pesquisa e Mestrado. Foi bolsista estadual (Governo do Estado do Pará / Universidade do Estado do Pará) durante o doutorado. Integra o Grupo de Pesquisa CUMA (Culturas e Memórias da Amazônia), coordenado pela Profa. Dra. Josebel Akel Fares da UEPA.

Ficha Catalográfica

Rodrigues, Irene Elias.

A inclusão de pessoas com necessidades educacionais especiais no processo educativo escolar: uma experiência inversa/ Irene Elias Rodrigues; Orientadora: Isabel Alice Osvald Monteiro Lelis; Co-orientadora: Oliveira, Ivanilde Apoluceno de. -2010.

196f. : il. (color.) ; $30 \mathrm{~cm}$

Tese (Doutorado em Educação) - Pontifícia Universidade Católica do Rio de Janeiro, Rio de Janeiro, 2010.

Inclui bibliografia

1. Educação - Teses. 2. Currículo. 3. Cultura escolar. 4. Diversidade cultural. 5. Literatura amazônica. 6. Cotidiano escolar. I. Lelis, Isabel Alice Osvald Monteiro. II. Oliveira, Ivanilde Apoluceno de. III. Pontifícia Universidade Católica do Rio de Janeiro. Departamento de Educação. IV. Título. 
Esta tese não poderia, sob nenhuma hipótese, ser dedicada a outras pessoas que não fossem aos meus pais Samuel e Elena, que sempre procuraram me repassar, a sua maneira, os diferentes ensinamentos dos valores humanos, hoje tão esquecidos pelas famílias. Apesar de semi-analfabetos, eles eram possuidores de um conhecimento singular que nenhuma escola oferece. Possuíam o conhecimento construído pela experiência de vida, estabelecida em uma hierarquia de valores rígidos em que a honestidade, o respeito, a dignidade, a verdade, a simplicidade e a fé, serviam de sustentáculo para qualquer decisão. Hoje ausentes, em outro plano, tenho certeza de que estão comemorando comigo mais uma vitória. Obrigada pai, obrigada mãe, vocês foram e serão, para mim, um grande exemplo de vida. 


\section{Agradecimentos}

$\mathrm{Na}$ construção de conhecimentos, toda ajuda é imprescindível. Às vezes, demoramos entender e reconhecer o significado da ajuda, por não termos a capacidade de perceber a forma como ela é emitida. Isso faz com que, muitas contribuições importantes, se percam no vazio da pseudo-sabedoria. Se acreditarmos que sabemos pouco, certamente estaremos em contínua busca de aprender, porém se pensamos que sabemos muito, acabamos desprezando contribuições valiosas, que poderiam fazer a diferença em nossa vida. Agradecer é um gesto de amor e de reconhecimento, entretanto é preciso cuidado no momento de elencarmos nomes aos nossos agradecimentos, pois é comum deixarmos de fora da lista, pessoas tão importantes e tão presentes, que parecem até já fazer parte de nossa história. Para não cair nesse erro, me limitarei a agradecer agrupando os diferentes segmentos e, agregado a eles, está inclusa cada pessoa que fez parte direta ou indiretamente da realização de mais este grande sonho - o doutorado. Agradeço:

À Pontifícia Universidade Católica do Rio de Janeiro, cujos professores me possibilitaram o acesso a uma diversidade de conhecimentos, que servirão de sustentação na minha prática docente.

À Universidade do Estado do Pará por fazer parte do DINTER e ter possibilitada a participação de seus professores no curso de doutorado.

À Escola Municipal de Ensino Fundamental Odinéa Leite Caminha que me permitiu adentrar na sua intimidade, disponibilizando todos os seus recursos (humanos e materiais) para que eu pudesse realizar a pesquisa que deu sustentação à tese. 
Aos meus colegas profissionais da Universidade do Estado do Pará, em especial do Núcleo de Tucuruí, que sempre me ajudaram nas horas mais difíceis em que a vontade de desistir era maior que a de prosseguir.

A todos os amigos que, de alguma forma, me ajudaram a tornar essa pesquisa cada dia mais estimulante, contribuindo com exemplos, reportagens, informações e até mesmo brincadeiras descontraídas nos momentos importantes da organização de idéias e pensamentos que fundamentaram esta tese.

À minha família, especialmente à minha filha Thulie Karime e aos meus netos Bárbara Karime, Pedro Henrique, Matheus Augusto, Pablo Filho e minha irmã Rosa que souberam me entender e me respeitar nos momentos de ausência, de aborrecimentos, de tédio, de solidão, de sofrimento, de choros e gargalhadas, sempre me incentivando a seguir em frente e acreditando na minha vitória.

À minha orientadora professora Isabel Alice Osvald Monteiro Lelis que se dispôs a enfrentar comigo esse grande desafio, sabendo o momento e a forma certa de cada passo e ação.

À minha co-orientadora professora doutora Ivanilde Apoluceno de Oliveira a quem devo a oportunidade de estar fazendo esse doutorado e que no colorido de suas orientações estabelecia com precisão onde e como eu poderia melhorar.

Finalmente o mais importante agradecimento: a DEUS que na sua infinita misericórdia me permitiu desfrutar do convívio de todas essas pessoas e me conduziu de forma sensata ao alcance de meu objetivo. Obrigada! 


\section{Resumo}

Rodrigues, Irene Elias. Lelis, Isabel Alice Osvald Monteiro. A inclusão de pessoas com necessidades educacionais especiais no processo educativo escolar: uma experiência inversa. Rio de Janeiro, 2010. 196. Tese de Doutorado Departamento de Educação, Pontifícia Universidade Católica do Rio de Janeiro.

Esta tese tem como objetivo principal analisar a trajetória da Escola Municipal de Ensino Fundamental Odinéa Leite Caminha, situada no município de Tucuruí, sudeste do Estado do Pará, unidade de ensino que se propôs a construir o processo de inclusão de forma diferenciada daquela prevista nos pressupostos legais: de instituição voltada para educação especial abriu-se para receber alunos do ensino regular, visando possibilitar o acesso à educação de qualidade para todos, indiscriminadamente. A realização da pesquisa teve como objetivos: identificar os procedimentos utilizados pela Escola Municipal de Ensino Fundamental Odinéa Leite Caminha, para sua transformação em escola de educação inclusiva, considerando que a prioridade inicial de seu atendimento era de educação especial; analisar como ocorreu o processo de inclusão dos alunos do ensino regular em uma escola de educação especial e qual a reação dos alunos antigos (educação especial), alunos novos (ensino regular) e da comunidade escolar frente à nova proposta de trabalho e identificar os avanços conquistados na e pela escola com a implantação do processo de inclusão e o nível de conhecimento dos diferentes segmentos sobre o referido processo. Para o alcance desses objetivos, os estudos realizados se pautaram nos motivos que conduziram a escola para essa iniciativa, nos procedimentos utilizados para sua transformação, a forma como ocorreram as mudanças e a expectativa dos alunos e da comunidade quanto à nova proposta de trabalho, além de pontuar quais os ganhos conquistados com a mudança bem como o nível de conhecimento da comunidade escolar sobre o processo de inclusão. $\mathrm{O}$ estudo realizado considerou os procedimentos didáticos antes e após a transformação e o atual funcionamento da escola considerando o currículo escolar, o projeto político pedagógico e o nível de relacionamento existente entre os diferentes segmentos envolvidos no processo. Para a realização do estudo, a metodologia utilizada foi a pesquisa qualitativa, que permitiu a análise das interações, a percepção de opiniões e a interpretação das 
especificidades de comportamentos e atitudes de cada partícipe de modo a possibilitar a indicação de contribuição para possíveis mudanças. Foi utilizado o estudo de caso, que permitiu detectar os motivos que sustentaram a decisão da mudança e os resultados obtidos durante todo o percurso do trabalho. Como suporte teórico para a pesquisa, foram utilizados vários documentos legais desde a constituição brasileira, legislações internacionais e nacionais que abordam o assunto bem como livros, artigos, textos (de diferentes autores), anotações de seminários e congressos, revistas e informações obtidas através dos meios de comunicação de massa e da própria internet. Para enriquecimento do estudo, foram realizadas quarenta entrevistas com representantes dos diferentes segmentos da escola (professores, funcionários, mães e alunos) além de vários contatos informais com registro no caderno de campo. Na análise das informações coletadas, foi possível identificar a prática pedagógica desenvolvida na e pela escola, o nível de relacionamento interpessoal, a flexibilidade curricular, a forma de utilização e confecção de materiais didáticos e o trato com a diversidade na ação educativa. Esses aspectos nos permitiram perceber a importância que os profissionais que atuam com a educação especial representam para a implantação do processo de inclusão, contribuindo tanto na ação docente com alternativas diferenciadas de trabalho quanto na sugestão de ações exequíveis para a operacionalização do processo de inclusão. Na construção do documento final - a tese - as entrevistas realizadas, os documentos escolares fornecidos para análise e as observações registradas no caderno de campo fortaleceram o registro possibilitando a realização de um documento mais preciso e capaz de propor sugestões tanto no âmbito escolar quanto na elaboração de políticas públicas norteadoras do processo de inclusão.

\section{Palavras-chave}

Inclusão; Processo educativo escolar; Educação Especial 


\section{Abstract}

Rodrigues, Irene Elias. Lelis, Isabel Alice Osvald Monteiro. Inclusion of special people with educational necessities in the process educative school: an inverse experience. Rio de Janeiro, 2010. 196. PhD thesis Departamento de Educação, Pontifícia Universidade Católica do Rio de Janeiro.

This thesis aims at analyzing the trajectory of the School of Basic Education Odinéa Leite Caminha, located in the city of Tucurui, southeastern of Pará, teaching unit which is proposed to construct the inclusion process in a different way from that anticipated in the legal assumptions: the institution devoted to special education has opened up for regular students, aiming to provide access to quality education for all, indiscriminately. The research aimed to identify the procedures used by the Municipal Elementary School Odinéa Leite Caminha, for its transformation into a school of inclusive education, whereas the initial priority of his attendance was special education, to analyze how the process occurred inclusion of regular students in a special education school and what the reaction of older students (special education), new students (regular education) and the school community in relation to new work proposal and identify the advances made in and around the school with the implementation of the inclusion process, and knowledge of the different segments on that process. To achieve these goals, the studies were based on motives that led the school for this initiative, the procedures used for processing, how the changes occurred and the expectation of students and community about the proposed new work, and which punctuate the gains with the change and the level of knowledge of the school community about the inclusion process. The study considered the didactic procedures before and after the transformation and the current operation of the school considering the school curriculum, the political pedagogical project and the level of relationship existing between the different parts involved in the process. To conduct the study methodology was based on qualitative research that allowed the analysis of interactions, the perception of opinion and interpretation of specific behaviors and attitudes of each participant to enable the indication of contribution to possible changes. It was also used the case study, which allowed to detect the reasons that supported the decision to change and the results obtained during the course of 
work. As technical support for the study used various legal documents from the Brazilian constitution, international and national laws that address the subject as well as books, articles, texts (from different authors), notes, seminars and conferences, magazines and information obtained through media mass communication and the Internet itself. For enrichment of forty interviews were conducted with representatives of different segments of the school (teachers, staff, students and mothers) and several informal contacts with record in field notebook. In the analysis of information collected could be identified in the pedagogical practice developed by the school and the level of interpersonal skills, flexibility, curriculum, how to use and making of teaching materials and dealing with diversity in educational activities. These features have allowed us to realize the importance that professionals who work with special education account for the deployment of the inclusion process, contributing in teaching activities with different alternatives of how much work the suggestion of feasible actions to operationalize the process of inclusion. In constructing the final document the interviews, school documents provided for review and comments recorded in the field notebooks have strengthened the study possible the realization of a more accurate and able to offer suggestions both in schools and in guiding public policy development the inclusion process.

\section{Keywords}

Inclusion; Educational process at school; Special Education 


\section{Sumário}

1. INTRODUÇÃO 17

2. INCLUSÃO: UM CAMINHO PARA CONSOLIDAÇÃO DA DEMOCRACIA

2.1. O Processo Histórico de Exclusão Social e Educacional de Pessoas com NecessidadesEducacionais Especiais 27

2.2. Política de Educação Inclusiva: da Normalização à Inclusão 36

2.2.1. O Princípio da Normalização 37

2.2.2. A Política de Integração 38

2.2.3. A Política de Inclusão 41

2.2.3.1. Política de Inclusão: Bases Legais 42

2.2.3.1.1. De Cunho Internacional 43

2.2.3.1.2. De Cunho Nacional $\quad 47$

2.2.3.2. Política de Inclusão: Pressupostos Pedagógicos 58

2.2.3.2.1. Adaptação Curricular 61

2.2.3.2.2. Processo de Avaliação 63

2.2.3.3. Política de Educação Inclusiva: Desafios 65

3. SISTEMA DE ENSINO E IMPLANTAÇÃO DA EDUCAÇÃO ESPECIAL 69

3.1. O Município de Tucuruí 69

3.2. Os Grandes Projetos $\quad 70$

3.2.1. A Estrada de Ferro Tocantins 70

3.2.2. A Usina Hidrelétrica $\quad 71$

3.3. O Sistema de Ensino 72

3.3.1. Implantação da Educação Especial $\quad 77$

3.3.2. Atendimento da Educação Especial pela Eletronorte 78

3.3.3. A Educação Especial sob a Gerência da

Secretaria Municipal de Educação 85

3.3.4. O Processo de Inclusão 93 
4. EDUCAÇÃO PARA TODOS: A TRAJETÓRIA DE

UMA ESCOLA DE EDUCAÇÃO ESPECIAL DE TUCURUÍ-PARÁ 96

4.1. A Escola Odinéa Leite Caminha 98

4.2. Aspectos Pedagógicos 106

4.2.1. Procedimentos de Seleção, Encaminhamento e Matrícula 106

$\begin{array}{ll}\text { 4.2.2. Enturmação } & 107\end{array}$

4.2.3. Projeto Político Pedagógico 111

4.2.4. Currículo 112

4.2.5. Avaliação 116

4.2.6. Formação Continuada 118

4.3. A Escola Odinéa e o Processo de Inclusão de Forma Inversa 122

5. A ESCOLA ODINÉA E A PRÁTICA DA EDUCAÇÃO INCLUSIVA 128

5.1. Sala de Aula: Espaço de Convivência e Aprendizagem 129

5.1.1. Procedimentos de Arrumação e Distribuição de Alunos 130

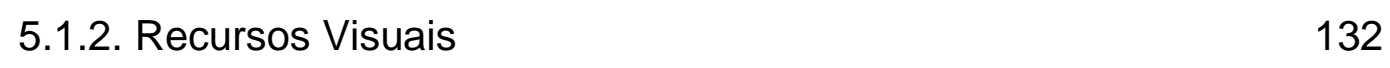

5.2. Metodologia de Trabalho 134

5.3. Prática Pedagógica 138

5.4. Relacionamento Interpessoal 146

$\begin{array}{ll}\text { 5.4. Atividades Complementares } & 151\end{array}$

6. CONSIDERAÇÕES FINAIS 166

$\begin{array}{lr}\text { 7. REFERÊNCIAS } & 176\end{array}$

APÊNDICE A - Roteiro de entrevista professor I 183

APÊNDICE B - Roteiro de entrevista professor II 185

APÊNDICE C - Roteiro de entrevista funcionários 187 
APÊNDICE D - Roteiro de entrevista pais

188

APÊNDICE E - Roteiro de entrevista alunos

190

ANEXOS

191 


\section{LISTA DE QUADROS}

Quadro 1 - Demonstrativo do número de escolas existentes no Município de Tucuruí.

Quadro 2 - Demonstrativo discente do Município de Tucuruí.

73

Quadro 3 - Demonstrativo da formação docente da rede municipal de ensino de Tucuruí.

Quadro 4 - Demonstrativo de alunos existentes na Educação Especial no ano em que a escola especial foi municipalizada.

Quadro 5 - Demonstrativo de atendimento na ed. especial no período 1993 - 1995

Quadro 6 - Demonstrativo de escolas com atendimento de alunos inclusos no município de Tucuruí em 2009.

Quadro 7 - Demonstrativo de alunos inclusos por categoria - ano 2009.

Quadro 8 - Demonstrativo do número de professores por modalidade de atendimento.

Quadro 9 - Demonstrativo de matrícula por turno/docente/não docente.

Quadro 10 - Quadro funcional da escola.

Quadro 11 - Situação sócio-econômica das famílias dos alunos.

Quadro 12 - Demonstrativo discente por turma e turno.

Quadro 13 - Correlação de atendimento de alunos por docente e não docente.

Quadro 14 - Demonstrativo de enturmação de alunos especiais por turno.

Quadro 15- Demonstrativo Acadêmico 2005 - 2009. 


\section{LISTA DE ABREVIATURAS E SIGLAS}

A - Aluno.

APAE - Associação de Pais e Amigos dos Excepcionais.

CCCC - Camargo Corrêa Comércio e Construção.

CEB - Câmara de Educação Básica.

CEE/PA - Conselho Estadual de Educação - Pará.

CENESP - Centro Nacional de Educação Especial.

CNE - Conselho Nacional de Educação.

COC - Colégio Osvaldo Cruz.

D - Diretor (a).

DA - Deficiência Auditiva.

DCN - Diretrizes Curriculares Nacionais.

DEEP - Diretoria de Estatísticas Educacionais.

DM - Deficiência Mental.

ECA - Estatuto da Criança e do Adolescente.

EFT - Estrada de Ferro Tocantins.

Eletronorte - Centrais Elétricas do Norte do Brasil S/A.

$\mathrm{F}$ - Funcionário.

IBGE - Instituto Brasileiro de Geografia e Estatística.

INEP - Instituto de Estudos e Pesquisas Educacionais Anísio Teixeira.

LDB - Lei de Diretrizes e Bases.

Libras - Língua Brasileira de Sinais.

M - Mãe.

MEC - Ministério de Educação e Cultura.

MW - Megawatts.

ONGS - Organizações não Governamentais.

ONU - Organização das Nações Unidas.

$P$ - Professor.

PAED - Programa de Complementação ao Atendimento Educacional

Especializado às Pessoas Portadoras de Deficiência.

PCN - Parâmetros Curriculares Nacionais.

PCNEM - Parâmetros Curriculares Nacionais para o Ensino Médio

PDDE - Programa Dinheiro Direto na Escola.

PDE - Plano de Desenvolvimento da Educação.

PNE - Pessoas com Necessidades Especiais.

PNEES - Pessoas com Necessidades Educacionais Especiais.

PPP - Projeto Político Pedagógico.

S - Supervisor (a).

SE - Secretaria de Educação.

SEB - Secretaria de Educação Básica.

SEESP - Secretaria de Educação Especial.

SEMDES - Secretaria Municipal de Desenvolvimento Econômico

Sustentável.

SEMED - Secretaria Municipal de Educação.

SFC - Sistema Floresta de Comunicação.

UHT - Usina Hidrelétrica de Tucuruí.

Unesco - Organização das Nações Unidas para a Educação, a Ciência e a Cultura. 
Pensar e viver a inclusão são um ato de amor que só pode brotar no coração das pessoas quando elas se dispõem a seguir o mandamento sagrado de "Amai-vos uns aos outros como EU vos amo". Se essa semente não germinar na vida das pessoas, sempre haverá a relação dispare entre o EU e o OUTRO impedindo a efetiva inclusão.

Irene Elias Rodrigues 